\title{
Dynamique spatio-temporelle d'occupation du sol dans la Réserve Totale de Faune de Tamou dans un contexte de la variabilité climatique (Ouest du Niger)
}

\author{
Barage MAMANE ${ }^{1,2^{*}}$, Garba AMADOU ${ }^{1}$, Moussa BARAGE ${ }^{1}$, Jacques COMBY ${ }^{2}$ et \\ Jean Marie Karimoune AMBOUTA ${ }^{1}$ \\ ${ }^{1}$ Université Abdou Moumouni de Niamey, Niger. \\ ${ }^{2}$ Université Jean Moulin Lyon III, France. \\ *Auteur correspondant; E-mail: mamanebarage44@gmail.com ; Tél: 0022790244898
}

\section{REMERCIEMENTS}

Les auteurs remercient l'Agence Universitaire de la Francophonie, l'administration du Parc National $W$ du Niger et le conservateur de la Réserve Totale de Faune de Tamou pour le partenariat grâce auquel ce travail de terrain a été financé.

\section{RESUME}

L'article traite de la dynamique spatio-temporelle des unités d'occupation du sol de la Réserve Totale de Faune de Tamou (RTFT), zone périphérique du Parc national W Niger, entre 1975 et 2013. L'objectif de cette étude est d'évaluer les mutations spatio-temporelles que connaît la RTFT à la suite des variations climatiques et des pratiques diverses observées en matière de l'exploitation des ressources. La méthode de numérisation visuelle qui consiste à délimiter les différentes unités homogènes a été appliquée. Les données utilisées sont trois (3) images satellitaires dont Landsat MSS de 1975, Landsat ETM de 1989 et Landsat ETM d'octobre 2013. Entre 1975 et 2013, la superficie des différentes unités d'occupation des terres a subi des mutations substantielles qui se traduisent par une régression des aires des formations forestières, une augmentation de celles des cultures pluviales et l'apparition de deux nouvelles unités qui sont les koris et les sols nus. Ces changements ont été faibles entre 1975 et 1989, mais plus marqués entre 1989 et 2013 avec l'apparition des nouvelles unités de sols nus et de koris en 2013, quasi inexistants entre 1975 et 1989. Ces deux dernières unités sont des indicateurs de forte dégradation du couvert forestier. Ainsi, les formations forestières qui occupaient $92,78 \%$ de la surface de la RTFT en 1975, sont passées de 87,62\% en 1989 à $68,54 \%$ de l'espace en 2013. Quant aux cultures pluviales qui occupaient 5,88\% de la surface en 1975, elles sont passées de $11,04 \%$ en 1989 à 28,25\% en 2013. La démographie galopante, l'agriculture, le surpâturage et les variations climatiques sont les principales causes de ces mutations.

(C) 2018 International Formulae Group. All rights reserved.

Mots clés: Occupation du sol, variabilité climatique, Parc National W du Niger, Réserve Total de Faune de Tamou, Dynamique, matrice de transition.

\section{Spatio-temporal dynamics of land use in the Total Tamou Fauna Reserve in a context of climatic variability (Western Niger)}




\section{ABSTRACT}

This work deals with environmental units trough spatiotemporal process from 1975 to 2013 of the total fauna reserve of Tamou TFRT, which is located across the Nigerien National Park border. Evaluate the spatiotemporal transformation recorded by TFRT following divers practices in terms of resources exploitation. Here we apply the digitalized visual methodology to delimitate the different homogeneous units. Therefore, we use three satellites images as database: the 1975 MSS Landsat, the 1989 ETM Landsat and October 2013 ETM Landsat. From 1975 to 2013 various occupational units areas were damaged. To $92.78 \%$ in 1989 , the estimated forest area is reduced to $87.62 \%$ in 1989 . So $43.04 \%$ of this area remained completely damaged. Besides that, $5.88 \%$ of the estimated pluvial culture increased to $11.04 \%$ in 1989. In the interval between 1989 and 2013 , we observed that the forested area have significantly receded to $68.54 \%$ in which $35.75 \%$ were deteriorated. On the other hand, the rainfed crops expanded and occupied $23.66 \%$ of the territory. Bare earth and koris nonexistent in 1975 and 1989, appeared and occupied respectively $0.60 \%$ and $2.89 \%$ in 2013. Population's growth, agriculture, pasturage, bush fires, and climatic variations are the main causes of these transformations.

(C) 2018 International Formulae Group. All rights reserved.

Keywords: Land cover, climate variability, Nigerien W Park, TFRT, Dynamic, Transition matrix.

\section{INTRODUCTION}

Les ressources naturelles de l'Afrique au sud du Sahara évoluent dans un environnement qui subit, à un rythme effréné et inquiétant, des transformations rapides. L'exploitation sans contrôle des écosystèmes forestiers de cette région imprime une forte dynamique d'occupation des sols et d'utilisation des terres. Cette dynamique qui se traduit en général par la dégradation du couvert végétal due principalement à l'intensification des activités anthropiques (Koffi et al., 2016) s'est largement amplifiée au cours de ces dernières décennies. Au Sahel, cette dégradation fait craindre que les maigres forêts disparaissent et/ou soient converties en terres agricoles à long terme si les pratiques culturales actuelles se maintiennent (Orékan et al., 2006a ; Oloukoi et al., 2006). Les écosystèmes forestiers constituent l'une des sources privilégiées de la population pour la satisfaction des besoins en produits, en l'occurrence la demande en nourriture, en bois d'œuvre et de service, en terres agricoles, etc. (Oswald et al., 2012 cité par Orékan et al ., 2014). Cette situation n'a pas épargné les aires protégées du Niger, un pays situé au cœur du sahel Ouest Africain. En effet, depuis quatre décennies, le Niger fait face à une dégradation continue de son environnement du fait des interactions de nombreux facteurs climatiques et anthropiques complexes. Alors que les 17129 076 d'habitants (INS, 2012) vivent essentiellement de l'agriculture pluviale, la baisse observée des précipitations moyennes depuis les années 1970, combinée à la variabilité inter annuelle et à une démographie galopante, ont entrainé plusieurs crises alimentaires nécessitant l'assistance de la communauté internationale. Ces sécheresses, combinées aux actions anthropiques, conduisent à la dégradation des ressources naturelles.

Devant une problématique aussi complexe, l'observation des paysages au cours du temps s'avère nécessaire afin d'estimer la nature et l'ampleur de l'évolution des processus naturels et anthropiques pour une gestion durable des ressources naturelles. L'analyse de la dynamique des formations forestières peut s'inscrire aussi dans le cadre des initiatives et stratégies d'atténuation des effets du changement climatique global. Aujourd'hui, l'utilisation de la télédétection et des données géo-spatiales pour la cartographie de l'occupation des terres est une activité courante des institutions intéressées par la gestion des ressources végétales (Inoussa et al., 2011a) . L'imagerie satellitaire joue un rôle incontournable dans le processus de caractérisations et d'aménagement des écosystèmes. Dans le Parc National W du Niger (PNWN), de nombreuses études 
adoptant des approches de télédétection et de SIG pour l'analyse de la dynamique d'écosystèmes ont été réalisées (Maman et al.,2011; Barbier et al., 2006; Clerici et al., 2007 ; Souley, 2010a). Cependant, les travaux spécifiques et actualisés sur la dynamique des unités paysagères qui protègent le noyau central du parc W sont insuffisants. La présente étude vise à combler cette lacune. Elle se fixe comme objectif général d'analyser les dynamiques d'occupation de l'espace et de dégradation du couvert végétal de la Réserve Totale de Faune de Tamou (RTFT). De façon spécifique, il s'agit de cartographier les unités d'occupation des sols dans la RTFT entre 1975,1989, et 2013 à partir des images Landsat et ressortir leurs dynamiques. Notre hypothèse de recherche est que dans la RTFT, la dynamique spatio-temporelle des unités d'occupations des sols au cours de ces trente (30) dernières années est liée à l'action anthropique dans un contexte de variabilité climatique.

\section{MATERIEL ET METHODES \\ Zone de l'étude}

Le Parc national $\mathrm{W}$ constitue un grand complexe régional écologique, s'étalant sur trois pays (Niger, Bénin et Burkina Faso) et couvrant une superficie totale de 1023000 hectares, dont 220000 hectares pour la partie nigérienne. Il est situé dans l'extrême sud- ouest du pays, entre les latitudes $11^{\circ} 54^{\prime}$ et $12^{\circ} 35^{\prime} \mathrm{N}$ et les longitudes $02^{\circ} 04^{\prime}$ et $2^{\circ} 50^{\prime} \mathrm{E}$. Il est bordé, entre autres, par la Réserve Totale de Faune de Tamou (RTFT: 77 167 ha). Concernant la RTFT (Figure 1), objet de la présente étude, elle a été créée par décret $n^{\circ}$ 62-188/MER du 8 août 1962 avec une superficie d'environ 147.000 ha. Celle-ci renferme presque les mêmes biotopes que le PWN et sert par conséquent de refuge à la faune de celui-ci à certaines périodes de l'année. Le parc est parcouru par trois principaux cours d'eau: le fleuve Niger et deux de ses affluents (Tapoa et la Mékrou). Les deux vents dominants dans cette zone sont l'harmattan, vent chaud et sec soufflant pendant toute la saison sèche et la mousson, vent humide, soufflant pendant la saison des pluies.
La RTFT appartient au système climatique tropical de type soudano-sahélien, dont le rythme des saisons est réglé par le déplacement du front intertropical (FIT) (Giannini et al., 2003). Le climat est caractérisé par une grande saison sèche (octobre-avril) et une courte saison pluvieuse (mai-septembre) caractérisée par une forte variation dans l'espace et dans le temps.

\section{Caractérisation du climat de la zone de l'étude}

Les données pluviométriques provenant de la Direction nationale de la météorologie du Niger (DNM) sont issues de sept stations (Niamey aéroport, Gaya, Birni-Gaouré, Tamou, Tapoa, Say et Falmey) pour la période (1960-2015) situées à l'intérieur et aux environs de la RTFT. Ces stations disposent de longues séries qui couvrent la période de l'étude (1975-2013). La mesure de l'évolution climatique a été conduite sur la base du calcul de l'Indice Standardisé de Précipitation (SPI) (Bergaoui et Alouini, 2001). Cet indice bien adapté au suivi des variations de la croissance de la végétation, est utilisé pour quantifier les déficits des précipitations à différentes échelles temporelles. Sa formule est la suivante : $\mathrm{SPI}_{\mathrm{i}}=(\mathrm{Xi}-\mathrm{Xm}) / \mathrm{Sm}$ où $: \mathrm{SPI}_{\mathrm{i}}$ : Indice Standardisé de Précipitation de l'année i $\mathrm{Xi}$ : est le cumul des pluies pour une année $\mathrm{i}$;

$\mathrm{Xm}$ et $\mathrm{Sm}$ sont respectivement la moyenne et l'écart type des pluies annuelles observés pour la série concernée. Le calcul de cet indice permet d'interpréter la dynamique de la couverture ligneuse en relation avec l'évolution de la pluviométrie (Koné et al., 2007a).

Lorsque le SPI > 2 on parle d'humidité extrême ;

Pour $1<$ SPI $<2$ l'humidité est forte ;

Pour $10<$ SPI < 1 l'humidité est modérée ;

Pour $-1<$ SPI $<0$ on parle de sécheresse modérée ;

$\mathrm{Si}-2<$ SPI $<-1$ la sécheresse est dite forte ;

Si SPI $<-2$ la sécheresse est qualifiée d'extrême.

\section{Démarche méthodologique Données utilisées}

L'ensemble de la zone du parc national W 
du Niger a pu être couvert par une mosaïque de scènes Landsat .Les images choisies (Tableau 1) sont acquises presque à la même période de l'année afin de réduire les problèmes liés aux différences d'angles solaires, au changement écologique de la végétation et à la différence d'humidité des sols. Toutes les images issues de la base des données du site www.Earth explorer.com sont acquises au niveau du Laboratoire d'Enseignement et de Recherche en Géomatique du Centre Régional AGRYMET à Niamey au Niger.

\section{Méthodes de traitement et d'analyse des Données : Cartographie de l'occupation du sol}

\section{Prétraitement des images satellitaires}

Tout d'abord, il faut noter que c'est seulement les canaux allant du visible au proche infra-rouge qui ont été utilisés dans cette étude (Canaux 1,2 et 3 pour le capteur MSS (Multi Spectral Scanner); canaux 2,3 et 4 pour le capteur TM (Thematic Mapper) et canaux 3,4 et 5 pour les capteurs OLI TIRS (Operational Land Imager), du fait des meilleures informations qu'ils apportent sur l'occupation des sols (Essifi, 2008; Bachir, 2013a). Des corrections radiométriques et atmosphériques sont faites sur ces différents canaux selon leurs degrés de perturbation. Une composition colorée (en fausse couleur) a été utilisée pour obtenir la meilleure visualisation des objets de l'image (Sarr, 2009).

\section{Classification numérique des images}

Le type de végétation a été identifié selon la typologie de Yangambi établie pour la cartographie de la végétation (Aubreville, 1957 modifié par De Wispelaere, 2002). De plus, les paramètres stationnels tels que le type d'unité géomorphologique, les états de surface du sol, la texture du sol, la présence de blocs de cuirasse, de blocs de grès, de gravillons, les termitières et autres traces d'activités anthropiques dont le passage du feu et le pâturage illégal ont été notés. Ces données ont permis d'améliorer et de valider la classification de la carte d'occupation des sols de 2013. L'identification des différentes unités a été faite sur la base d'une clé d'interprétation des photographies aériennes définies dans une zone du Sahel (NOS Niger 2001). Compte tenu de la connaissance du terrain, l'étude s'est basée sur la numérisation et la classification visuelle à l'écran. Cette technique qui s'apparente à la classification supervisée consiste à numériser manuellement et de façon exhaustive toutes les unités homogènes des images fausse couleur de la zone d'étude et de procéder à leurs classifications. Ce choix s'explique du fait que certaines classes d'utilisation et d'occupation des terres, telles que les cultures au Sahel, n'ont pas pu être distinctement différenciées des autres classes sur la base de leurs propriétés spectrales. Les méthodes de classifications automatiques d'images satellites sont basées sur la différenciation des valeurs spectrales des pixels des images et révèlent souvent des erreurs de classifications. La réflectance spectrale des surfaces terrestres mesurée par les capteurs de télédétection est une valeur quantitative qui n'est pas indiscutable (Bachir, 2013b). En effet, il existe une forte variabilité au sein des valeurs de réflectance spectrale associées à divers types d'occupation des terres (Lillesand and Kiefer, 1994). Cette variabilité pose des problèmes majeurs au niveau de la cartographie et de l'analyse des types d'occupation des terres basées sur leurs seules propriétés spectrales.

\section{Traitement post-classification et validation des résultats}

La validation de cette classification a nécessité une mission de vérité terrain qui a permis de vérifier sur le terrain un certain nombre de points pris au niveau des différentes unités d'occupation des sols de la carte 2013 provisoire. Ce qui a permis enfin d'affiner la précision de cette carte. Ainsi les cartes définitives ont été générées à la fin de cette mission. Le processus du traitement des images est synthétisé dans la Figure 2.

\section{Détection des changements \\ Evolution de l'occupation du sol}

Pour l'étude de la dynamique des ressources naturelles, une étude diachronique des images satellitaires de 1975, 1989 et 2013 a été réalisée. Cette étude a consisté 
à mettre en évidence les transformations survenues entre deux périodes suite au croisement des cartes de 1975, 1989 et 2013. Après la vectorisation automatique, les résultats de la classification sont exportés en format vectoriel pour les traitements cartographiques à l'aide d'un logiciel Arc GIS 10.3 .

Le croissement de trois cartes d'occupation du sol de 1975, 1989 et de 2013 a permis d'établir la carte d'évolution de toutes les unités paysagères durant ces trente-huit (38) années. Ce croisement a été réalisé en se fondant sur une codification des classes d'unité d'occupation de sol (Tableau 2) et une comparaison des codes entre les trois cartes. Il s'agit d'une approche de vérification des changements à partir des changements de codes pour les vecteurs homologues.

\section{Analyse statistique}

Quantification du changement : Afin de quantifier les changements au niveau des classes d'occupation du sol, plusieurs indicateurs statistiques ont été calculés ; il s'agit des taux de changement et de la matrice de transition.

Matrice de transition : Ainsi, pour décrire ces changements d'occupation du sol intervenus entre 1975 et 1989 , entre 1989 et 2013 et enfin entre 1975 et 2013, des statistiques descriptives sur les superficies de différentes unités d'occupation du sol pour les trois périodes ont été faites. Le croisement à l'aide du module « geoprocessing » d'ArcGIS 10. 3 des trois cartes d'occupation du sol a permis d'obtenir une matrice de transition qui a renseigné sur les mutations spatiales des classes au cours des intervalles 1975-1989,19892013, 1975-2013. Les matrices de confusion confrontant les différentes classes thématiques ont servi pour la vérification de la performance des classifications.

Les logiciels ENVI5.1, ArcGIS10.3 et QGIS 2.14 ont servi dans le traitement des images satellitaires (pour le premier), la création des cartes thématiques (pour le second) et la production /extraction des statistiques de zones (pour le troisième).

Elle correspond à une matrice carrée décrivant de manière condensée, les changements d'état des classes thématiques pendant la période considérée (Bamba et al., 2008). Les valeurs de colonnes et des lignes représentent les surfaces d'occupation $\mathrm{du}$ sol pour chaque classe à la date correspondante. Les colonnes de la matrice indiquent les états d'occupations des sols en 2013 et les lignes correspondantes aux états en 1975. Pour apprécier le processus de modification, des vecteurs (shapefile) ont été créés à un temps T0 ou année N0 délimitant le contour et la superficie de chaque unité paysagère contenue dans la zone de l'étude.

Après superposition des fichiers de formes de l'année $\mathrm{N}$ et $\mathrm{N} 0$ on procède à l'extraction en fonction des unités paysagères sur les espaces occupés par ces unités de la partie de l'unité paysagère à l'année NO.Le fichier de forme issu de ce découpage représente la dérivation de l'unité (année N0) à l'année N.

Ensuite la proportion de chaque unité dans la dérivation a été calculée par le processus suivant : Le nombre de polygones est l'un des paramètres les plus importants pour décrire le paysage. Pour une classe, le nombre total de polygones est la somme des polygones qui composent cette classe. Quant à l'aire totale (exprimée en hectares) d'une classe $\mathrm{j}\left(\mathrm{S}_{\mathrm{j}}\right)$, c'est la surface totale occupée par l'ensemble de ses polygones i (3). $\mathrm{S} \mathrm{j}=\Sigma \mathrm{s}_{\mathrm{i}}$ Avec $\mathrm{s}_{\mathrm{i}}=$ surface occupée par un polygone (en ha). $\mathrm{i}=1 \ldots \mathrm{n}$.

\section{Calcul des taux de changement}

Après l'établissement des cartes d'occupation des terres de 1975 (T1), 1989 (T2) et 2013 (T3), une analyse comparative de trois états $\mathrm{T} 1, \mathrm{~T} 2$ et $\mathrm{T} 3$ a été faite. Le bilan d'évolution de l'occupation du sol ainsi que le taux de changement entre les surfaces des unités d'occupation des terres entre les dates 1975 -1989 (T1-T2), les dates 1989-2013 (T2- T3) et les dates 1975-2013 (T1-T3) ont été déterminés. L'évaluation des changements est quantifiée au moyen de la 
formule proposée par la FAO (1996) et dont l'utilisation est très courante (NoyolaMedrano, 2009). Elle s'établit comme suit : $\mathrm{T}=(1 /(\mathrm{t} 2-\mathrm{t} 1)) * \ln (\mathrm{S} 2 / \mathrm{S} 1)$ avec $\mathrm{S} 1$ et $\mathrm{S} 2$ correspondant respectivement aux superficies d'une catégorie d'occupation du sol en année $\mathrm{t} 1$ et en année $\mathrm{t} 2 ; \mathrm{T}=$ taux de changement; ln le logarithme népérien.
Les valeurs positives du bilan représentent une progression de la surface de la classe pendant la période analysée et les valeurs négatives indiquent la perte de surface (régression) d'une classe entre les deux dates. Les valeurs proches de Zéro indiquent que la classe reste relativement stable entre les deux dates.

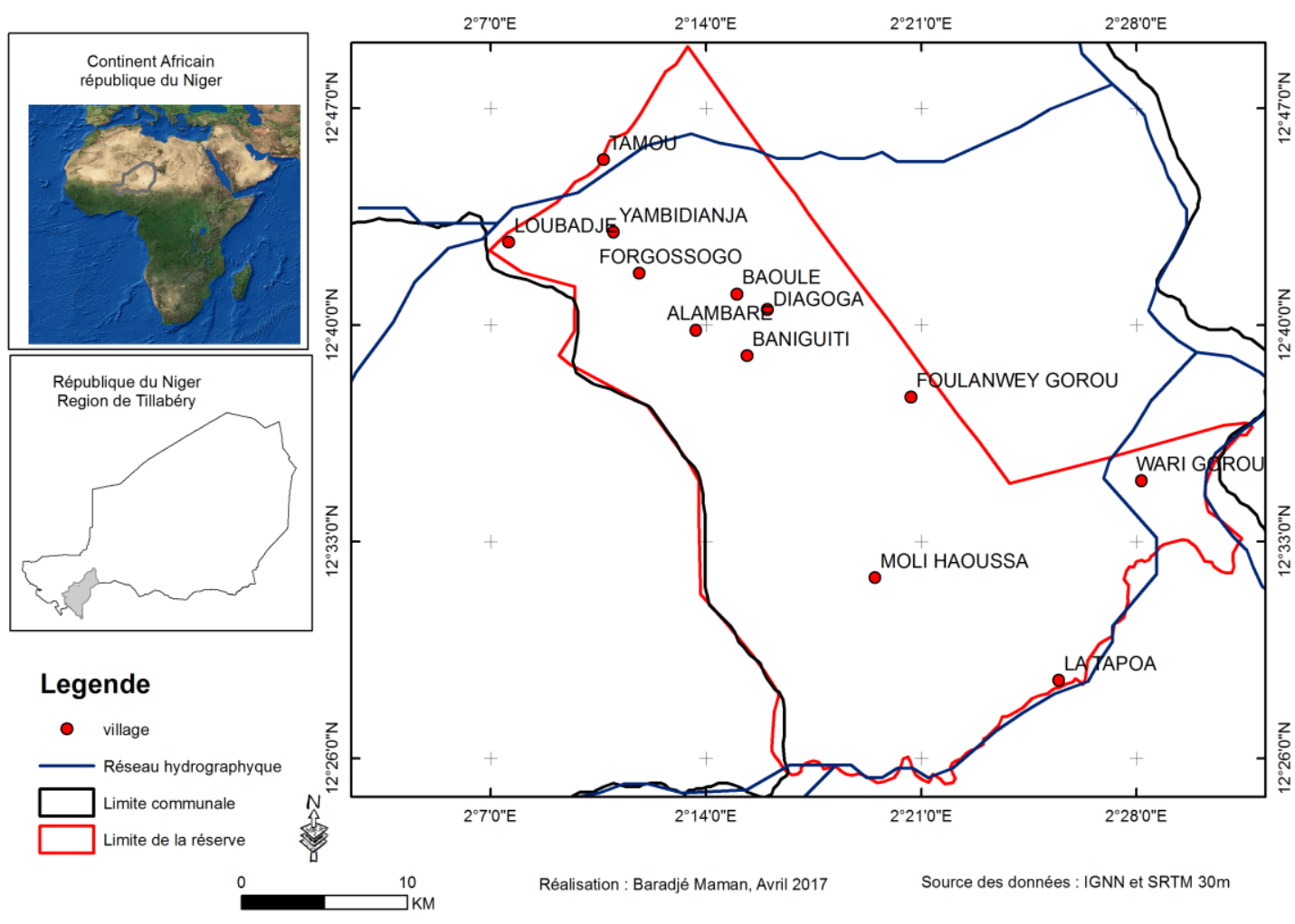

Figure 1 : Carte de localisation de la RTFT.

Tableau 1 : Récapitulatif des images utilisées pour les différentes périodes.

\begin{tabular}{llllccc}
\hline \multicolumn{1}{c}{ Satellite } & Capteurs & Path & Row & Résolution $(\mathbf{m})$ & & Date \\
${$\cline { 1 - 3 } $2-3} }$ & MSS & 207 & 51 & 60 & & 22 novembre 1975 \\
Landsat 4 & TM & 193 & 51 & 30 & & 20 novembre 1989 \\
Landsat8 & OLI TIRS & 193 & 51 & 30 & & 12 octobre 2013 \\
Landsat8 & OLI TIRS & 192 & 51 & 30 & & 21 octobre 2013. \\
\hline
\end{tabular}




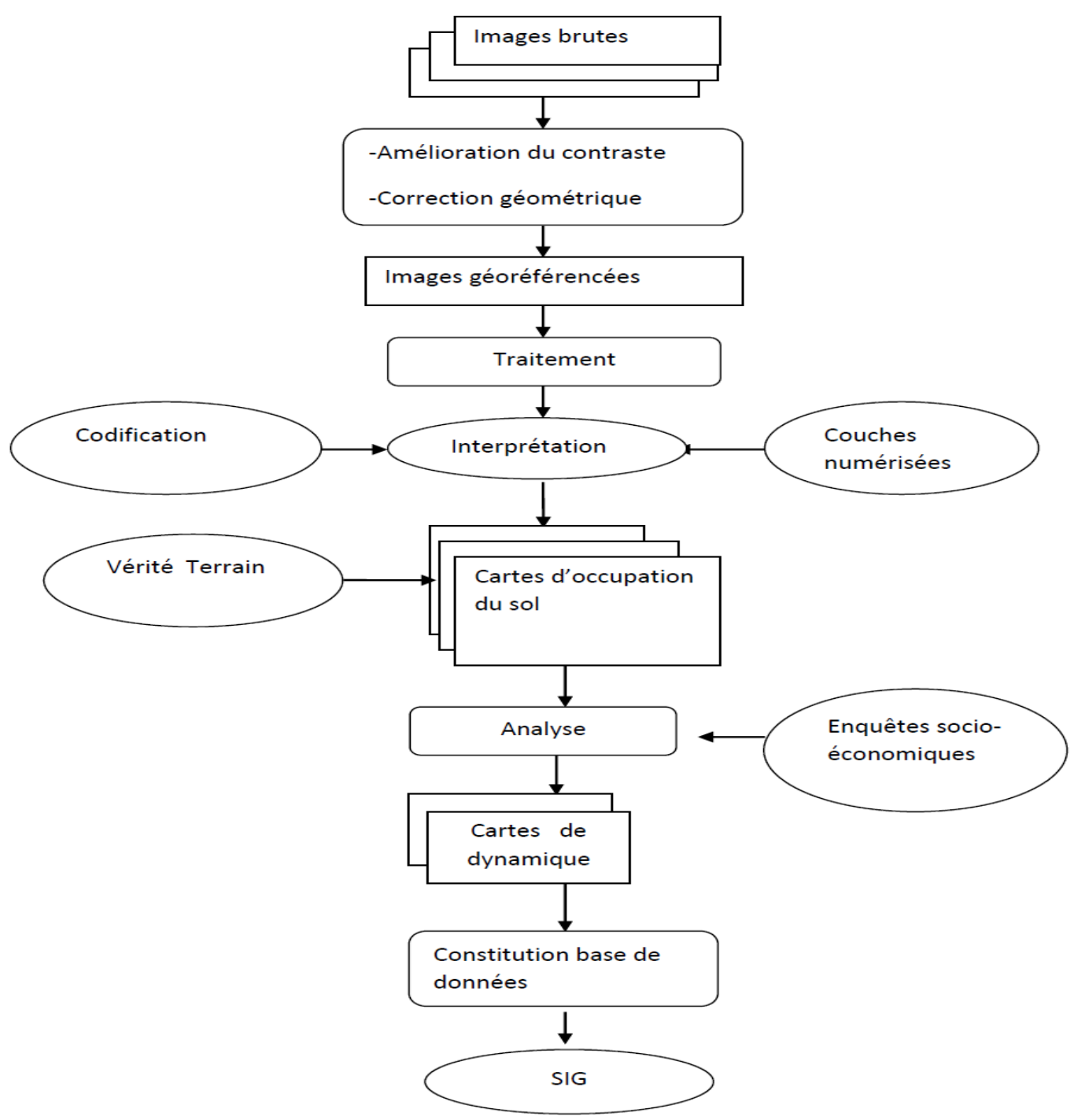

Figure 2 : Synthèse des traitements des images.

Tableau 2 : Unités d'occupation du sol.

\begin{tabular}{ll}
\hline Classes d'occupation du sol & Description \\
\hline Affleurement rocheux & Ce sont des cuirasses rocheuses. \\
Brousse tachetée dégradé & $\begin{array}{l}\text { Une composition floristique plus pauvre } \\
\text { Cultures pluviales }\end{array}$ \\
Forêt claire & $\begin{array}{l}\text { Un peuplement multi strate à feuille caduques, arbres de taille } \\
\text { moyenne et à cimes sont jointives (60-80\%). }\end{array}$ \\
Forêt claire dégradée & $\begin{array}{l}\text { Peuplement multi-strates dans laquelle le sous-bois est disparu } \\
\text { Forêt galerie }\end{array}$ \\
Koris & $\begin{array}{l}\text { Une incision dont le lit porte des alluvions grossières (sable, } \\
\text { graviers). }\end{array}$ \\
Savane arbustive & $\begin{array}{l}\text { Une formation d'arbuste (recouvrement entre 5 et 25\%) à } \\
\text { tapis herbacé. }\end{array}$ \\
\hline
\end{tabular}




\section{RESULTATS}

\section{Evolution climatique}

L'analyse du graphique des anomalies standardisées des données des précipitations moyennes (Figure 3) recueillies au niveau de sept stations sus indiquées au cours de la période (1960-2015) révèle une alternance entre les années de sécheresses et les années humides avec toutefois des épisodes secs de plus en plus longs. La décennie 60 se caractérise par une pluviosité excédentaire par rapport à la normale 1961-1990 tandis que la pluviosité des décennies 70 et 80 est globalement déficitaire. A partir de la décennie 90, la pluviosité de la zone se caractérise par des années excédentaires mais souvent avec des années déficitaires.

Les données observées sur la température de 1960 à 2015 (Figure 4) montrent une tendance nette à la hausse. Les décennies 90 et 2000 sont les décennies les plus chaudes. Depuis 1980, la tendance quinquennale des températures maximales est à la hausse. L'alternance rapprochée entre années sèches et humides additionnée à l'augmentation des températures a eu des impacts négatifs sur les ressources naturelles de la zone périphérique du parc $\mathrm{W}$ du Niger.

\section{Etat de l'occupation des sols 1975, 1989 et 2013}

A l'issue du traitement des images des données collectées sur le terrain et à l'usage des anciennes cartes topographiques, neuf unités d'occupation des sols ont été distinguées comme l'illustrent les Figures 5, 6 et 7 en s'inspirant de la classification des formations végétales de 1956 à Yangambi.

La Figure 5 présente la carte d'occupation du sol de la RTFT en 1975. Elle est réalisée à partir de l'interprétation de la photographie aérienne Landsat MSS du 22 novembre 1975. Il est observé une prédominance des forêts claires couvrant globalement $80,06 \%$ de la superficie totale dont $24,33 \%$ sont dégradées et sont réparties du nord au sud de la RTFT. Cependant, les forêts claires dégradées sont nettement localisées aux alentours des villages de Foulanwey Gorou, Wari Gorou et Moli Haoussa. La brousse tachetée dégradée est estimée à $4,16 \%$ de la superficie totale. Quant aux forêts galeries, elles occupent $8,56 \%$ et sont étendues sur le long des cours d'eau la Tapoa au sud. Les zones de cultures occupent $5,88 \%$ de la superficie totale. A cette époque, elles sont situées principalement au nord de la RTFT à côté du village de Tamou. Les effleurements rocheux occupent $1,34 \%$ et sont situés complètement à l'est.

La Figure 6 présente la carte d'occupation du sol de la RTFT en 1989. A cette période, les affleurements rocheux restent inchangés par rapport à 1975 avec 1,34\% et les formations des forêts claires couvrent globalement $67,7 \%$ dont $31,20 \%$ de forêts claires dégradées. La forêt galerie couvre $7,34 \%$ de cette superficie. Les brousses tachetées dégradées représentent $6,04 \%$. La savane arbustive couvre $6,54 \%$ et les cultures pluviales $11 \%$.

La Figure 7 présente les superficies des unités d'occupation du sol dans la RFT en 2013. Les étendues des cultures pluviales $(28,25 \%)$ sont présentes un peu partout dans la RTFT sauf au sud mais dominantes surtout au nord. La brousse tachetée dégradée est estimée à 4,62\%, et elle devient de plus en plus importante toujours dans le nord. Les forêts claires et les forêts claires dégradées représentent respectivement $21,37 \%$ et $35,65 \%$ et elles sont concentrées au sud. La forêt galerie est estimée à 6,79\% et occupe toujours le long de la rivière Tapoa, et la savane arborée est de $0,81 \%$. Les nouvelles unités d'occupations font leur apparition : les Koris et les sols nus représentant respectivement $5,65 \%$ et $0,81 \%$.

\section{Analyse de l'évolution de l'occupation des sols entre 1975 et 1989 dans la RTFT}

L'évaluation de cette évolution permet de mettre en évidence les changements qui sont intervenus durant la période 1975 - 1989. Durant cette période on note une tendance à la régression des superficies des forêts claires, des forêts galeries, qui passe respectivement de $55,7 \%$ à $36,5 \%$ et de $8,6 \%$ à $7,3 \%$. Au même moment, les surfaces de la brousse tachetée dégradée, les cultures pluviales, la forêt claire dégradée, la savane arbustive voient leur superficie augmenter respectivement de $4,2 \%$ à $6 \%$; de $5,9 \%$ à $11 \%$ et de $24,3 \%$ à $31,2 \%$. La savane 
arbustive, pratiquement inexistante en 1975, a occupé 6,5\%. L'évolution des différentes unités d'occupation du sol durant la période 1975-1989 est présentée par le Tableau 3.

La matrice de transition révèle que seuls 1031 ha des affleureusements rocheux sont restés inchangés pour la période (19751989) alors que les autres unités d'occupation du sol ont subi une conversion vers d'autres classes (Tableau 4). Celle de la dynamique en superficie des unités d'occupation des sols de la RTFT entre 1989 et 2013 est présentée par le Tableau 5. On observe à travers ce Tableau 5 la façon dont toutes les unités d'occupations ont subi des changements tant dans leur superficie que dans leur nature pendant la période 1975 à 1989 à l'exception des affleurements rocheux restés stables. Le Tableau 5 extrait de la Figure 6 met en relief les principales évolutions survenues entre 1989 et 2013 dans les unités d'occupation des sols.3.

L'analyse du Tableau 6 révèle que sur les neufs types d'unités d'occupation du sol dans la RTFT, trois unités ont connu une régression relativement importante, ce qui représente $30,9 \%$ de la superficie totale de la RTFT. Il s'agit de la forêt claire, la forêt galerie et la brousse tachetée dégradée. Par ailleurs, trois unités ont connu une progression très importante. Cette augmentation est estimée à environ 28575 ha soit $67,2 \%$ de la superficie totale de la RTFT et concerne les unités d'occupations suivantes: la culture pluviale, la forêt claire dégradée et la savane arbustive. Durant cette même période les affleurements rôcheux sont restés stables et d'autres nouvelles unités ont fait leur apparition comme les koris et le sol nu qui n'existaient pas en 1975. La synthèse de cette évolution nous montre que les formations naturelles sont passées de 68386 à 47948 ha, soit une régression de 20438 ha qui ont subi des conversions en d'autres unités d'occupations, ces différentes mutations ont été décrites au niveau de la matrice de transition du Tableau 7. Les formations anthropisées ont connu une augmentation de leur superficie passant de 4536 à 21802 hectares soit 6,5\% d'accroissement. Mais, il faut souligner que les formations naturelles représentent encore $62,1 \%$ de la superficie totale de la RTFT, contre $36 \%$ pour les formations anthropisées. Ainsi, il y'a un recul généralisé des forêts sur l'ensemble de la RTFT et une forte progression des surfaces cultivées. Toutefois, l'évolution des unités d'occupation du sol n'est pas totalement identique dans toute la RTFT. La Figure 8 permet de mieux visualiser la tendance d'évolution des cultures pluviales de 1975, 1989 et 2013. La rareté de nouvelles terres à défricher dans la zone «Ayinoma» (Mot Haoussa qui signifie cultiver la terre) déclassée en 1978 a conduit à l'exploitation de la RTFT.

La matrice de transition (1975 - 2013) sous forme de (Tableau 7) ci-dessus illustre la dynamique des unités paysagères de la RTFT .Par rapport aux ; affectations d'occupation des sols, les 3904 ha occupent la brousse tacheté dégradée en 1975 sont trasnformés de la manière suivante $(3617,90$ ha sont devenus cultures pluviales, $68 ; 13$ ha sont devenus savane arbustive; 38,57 ha sont devenus sol nu et 179, 41 ha sont restés inchangés toujours brousse tachétée dégradée). De même, les superficies initiales de forêts galeries $(6944,26$ ha) en 1975 ont subi les transformations suivantes $(6771,50$ ha en cultures pluviales; 2,28 ha en koris ; 0,48 en sol nu ; 47,61 en forêts claires dégradées , 119,71 ha sont restés inchangés). L'analyse synthétique des modifications subies par la RTFT entre 1975 et 2013 permet de comprendre l'importance des dynamiques paysagères partitionnées de la façon suivante : l'ouverture du milieu $(28,25$ $\%$ du paysage) se caractérise par une extension de cultures pluviales de la périphérie vers la zone intégralement protégée de la RTFT, la réduction des forêts claires $(51,13 \%$ de forêts claires 1975) et $26,70 \%$ de forêts claires, en 2013) au profit des forêts claires dégradées $(27,43 \%$ de forêts claires dégradées en 1975) $(34,54 \%$ de forêts claires dégradées en 2013). Par ailleurs on constate une diminution drastique des forêts galeries (qui étaient $9 \%$ en 1975 et $0,9 \%$ en 2013).

La Figure 8 ci-dessous présente la synthèse de l'évolution de toutes les unités paysagères notamment les cultures pluviales de 1975 à 2013 dans la RTFT. 


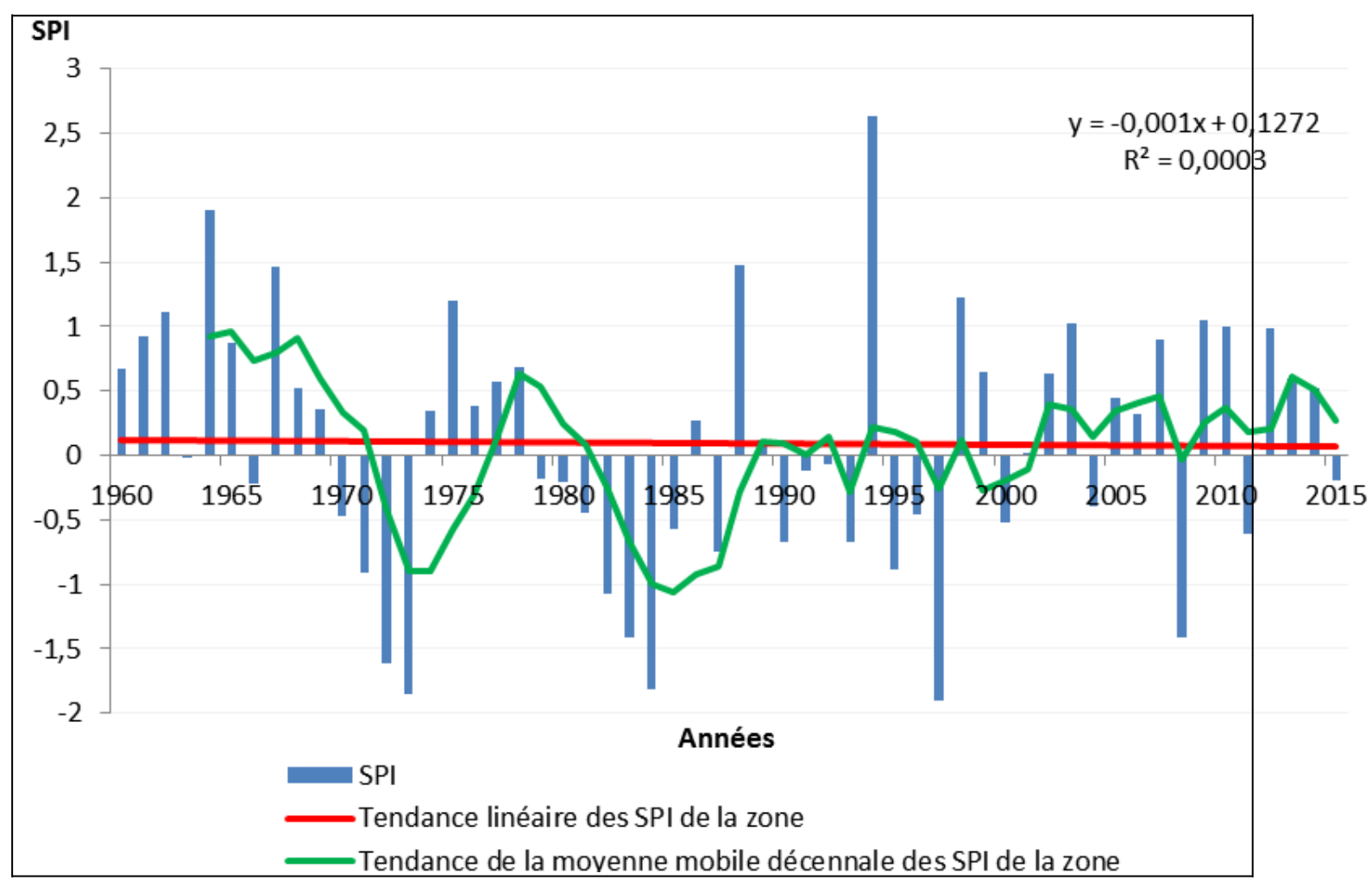

Figure 3 : Variation de l'Indice Standardisé des précipitations moyennes annuelles dans la zone périphérique du parc W du Niger (1960-2015).

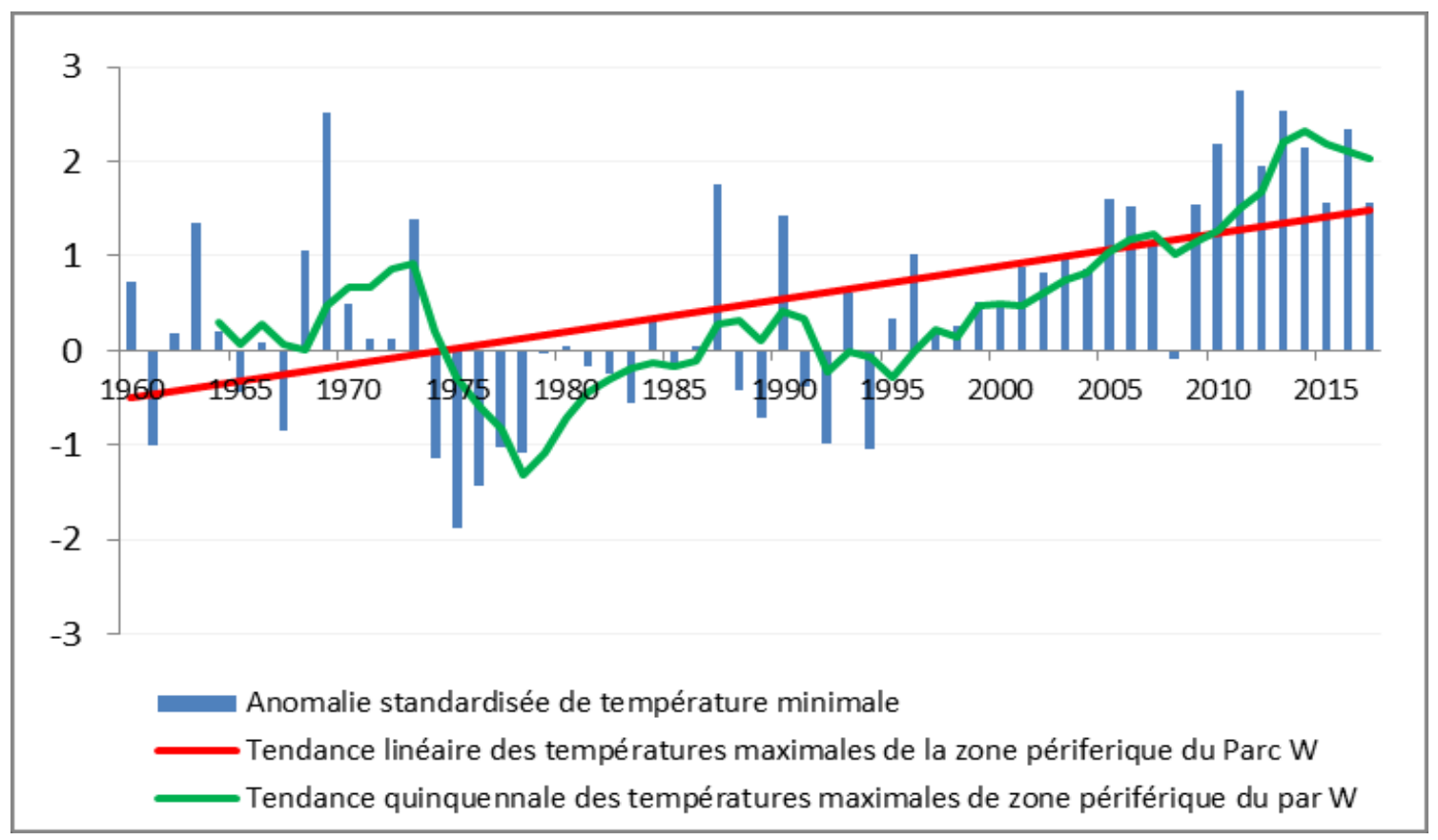

Figure 4 : Variation de l'Indice Standardisé de températures moyennes mensuelles dans la zone périphérique du parc W du Niger (1960-2015). 


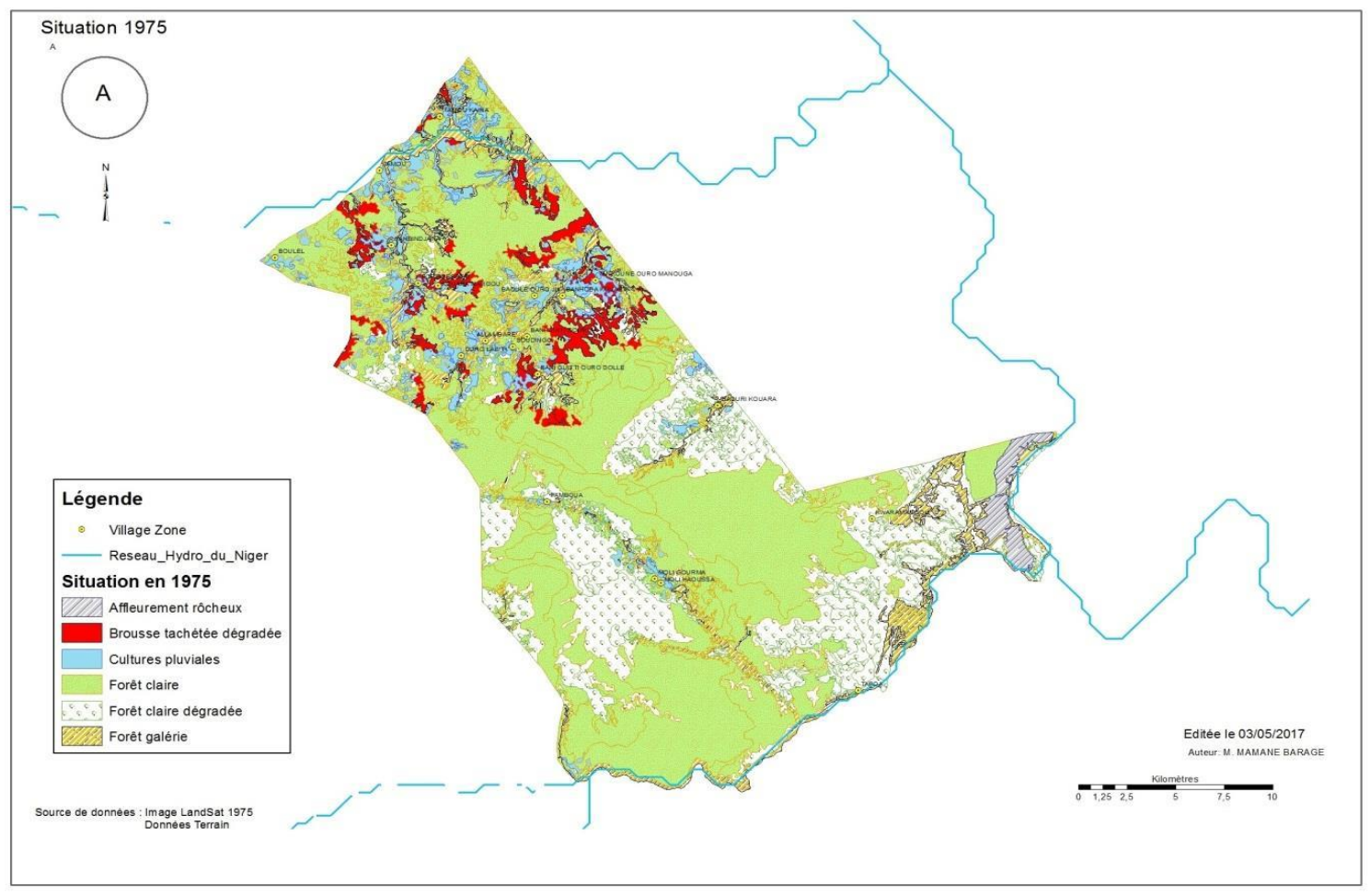

Figure 5: Carte d'occupation des sols en 1975 dans la RTFT.

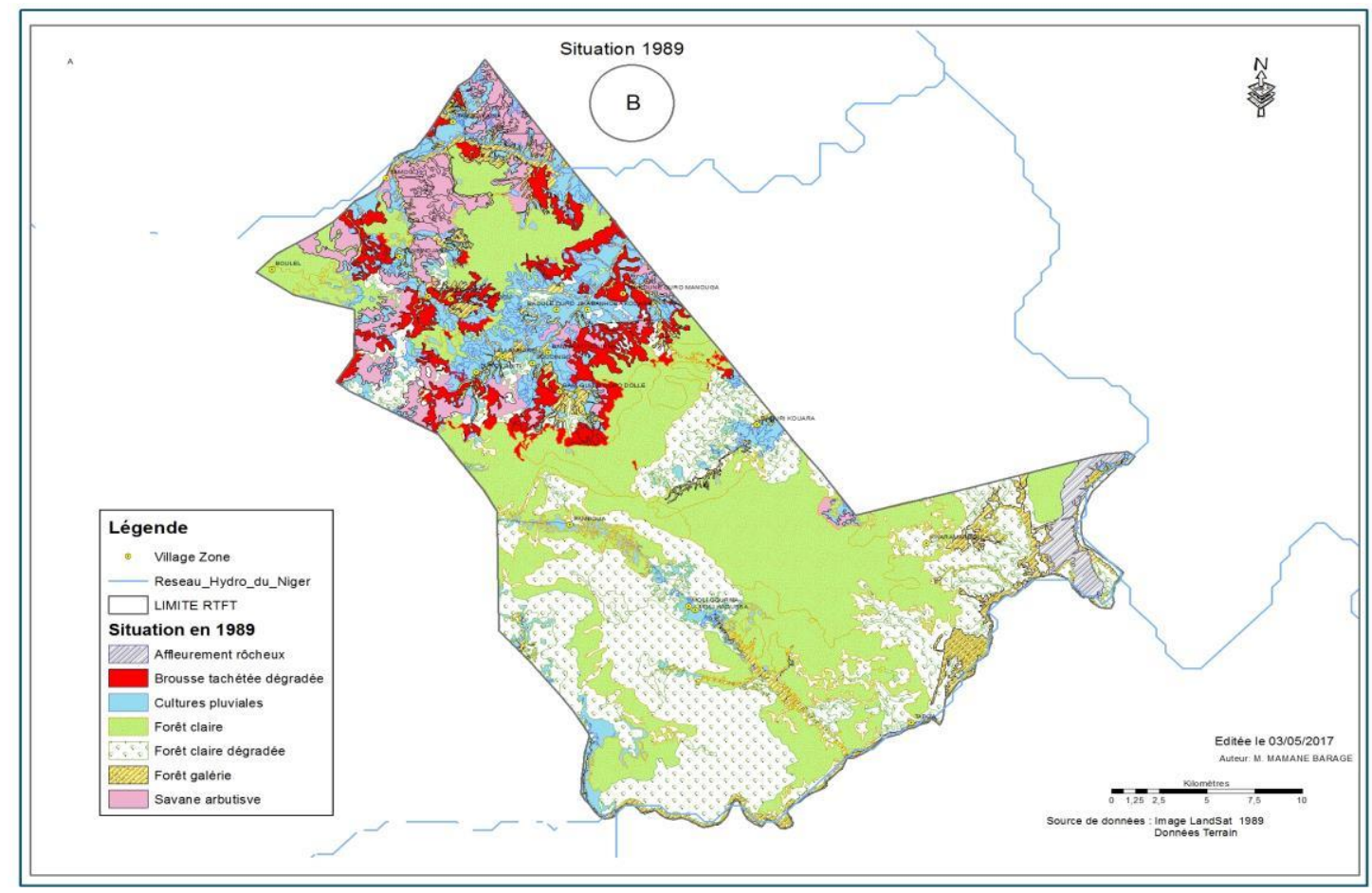

Figure 6 : Carte d'occupation des sols en 1989 dans la RTFT. 


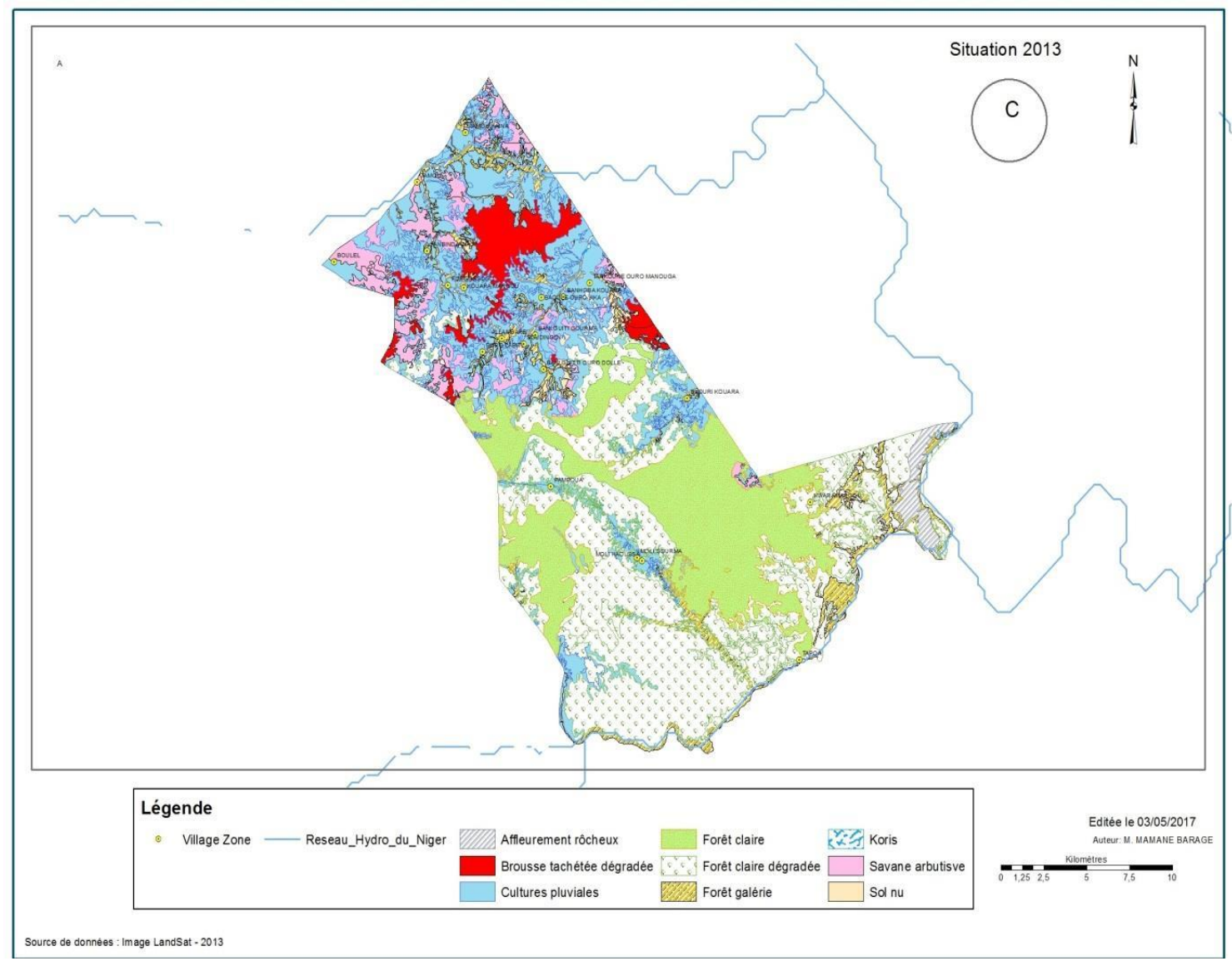

Figure 7 : Carte d'occupation des sols en 2013 dans la RTFT.

Tableau 3 : Evolution des différentes unités d'occupation des sols de 1975 à 1989.

\begin{tabular}{|c|c|c|c|c|c|c|c|}
\hline \multirow{2}{*}{$\begin{array}{l}\text { Type } \\
\text { d'occupation }\end{array}$} & \multicolumn{2}{|c|}{ Etat en 1975} & \multicolumn{2}{|c|}{ Etat en 1989} & \multirow{2}{*}{$\begin{array}{l}\text { Progression } \\
\text { 1975- } 1989 \text { (ha) }\end{array}$} & \multirow{2}{*}{$\begin{array}{l}\text { Régression en } \\
1975-1989 \text { (ha) }\end{array}$} & \multirow{2}{*}{$\begin{array}{l}\text { Taux de } \\
\text { Cnangement en } \\
\text { 1975-2013 (\%) }\end{array}$} \\
\hline & ha & $\%$ & ha & $\%$ & & & \\
\hline $\begin{array}{l}\text { Affleurement } \\
\text { rôcheux }\end{array}$ & 1031 & 1,3 & 1031 & 1,3 & & & 0,0 \\
\hline $\begin{array}{l}\text { Brousse tachetée } \\
\text { dégradée }\end{array}$ & 3214 & 4,2 & 4665 & 6 & 1451 & & 1,6 \\
\hline Cultures pluviales & 4536 & 5,9 & 8523 & 11 & 3987 & & 2,6 \\
\hline Forêt claire & 43008 & 55,7 & 28166 & 36,5 & & -14842 & $-1,8$ \\
\hline $\begin{array}{l}\text { Forêt claire } \\
\text { dégradée }\end{array}$ & 18774 & 24,3 & 24072 & 31,2 & 5298 & & 1,0 \\
\hline Forêt galerie & 6604 & 8,6 & 5667 & 7,3 & & -937 & $-0,6$ \\
\hline Savane Arbustive & & 0,0 & 5043 & 6,5 & 5043 & & \\
\hline TOTAL & 77167 & 100 & 77167 & 100 & & & \\
\hline
\end{tabular}


Tableau 4: Dynamique des unités d'occupation des sols en hectare dans la RTFT entre 1975 et 1989.

\begin{tabular}{|c|c|c|c|c|c|c|c|c|}
\hline $\begin{array}{l}\text { Classes } \\
\text { d'occupation du } \\
\text { sol }\end{array}$ & $\begin{array}{l}\text { Affleurement } \\
\text { rôcheux }\end{array}$ & $\begin{array}{l}\text { Brousse } \\
\text { tachétée } \\
\text { dégradée }\end{array}$ & $\begin{array}{l}\text { Cultures } \\
\text { pluviales }\end{array}$ & $\begin{array}{l}\text { Forêt } \\
\text { claire }\end{array}$ & $\begin{array}{l}\text { Forêt claire } \\
\text { dégradée }\end{array}$ & $\begin{array}{l}\text { Forêt } \\
\text { galerie }\end{array}$ & $\begin{array}{l}\text { Savane } \\
\text { arbustive }\end{array}$ & $\begin{array}{l}\text { Total } \\
1975\end{array}$ \\
\hline $\begin{array}{l}\text { Affleurement } \\
\text { rôcheux }\end{array}$ & 1031 & - & - & - & - & - & - & 1031 \\
\hline $\begin{array}{l}\text { Brousse tachetée } \\
\text { dégradée }\end{array}$ & - & 1501 & 97 & 1602 & - & - & 14 & 3214 \\
\hline $\begin{array}{l}\text { Cultures } \\
\text { pluviales }\end{array}$ & - & 1203 & 1101 & 1378 & 235 & 18 & 601 & 4536 \\
\hline Forêt claire & & 1774 & 6556 & 15993 & 13848 & 811 & 4026 & 43008 \\
\hline $\begin{array}{l}\text { Forêt claire } \\
\text { dégradée }\end{array}$ & -- & 91 & 190 & 7362 & 9522 & 1287 & 322 & 18774 \\
\hline Forêt galerie & - & 96 & 577 & 1831 & 467 & 3551 & 82 & 6604 \\
\hline Total 1989 & 1031 & 4665 & 8521 & 28166 & 24072 & 5667 & 5043 & 77167 \\
\hline
\end{tabular}

Tableau 5: Dynamique des unités d'occupation du sol en hectare dans la RTFT entre 1989 et 2013.

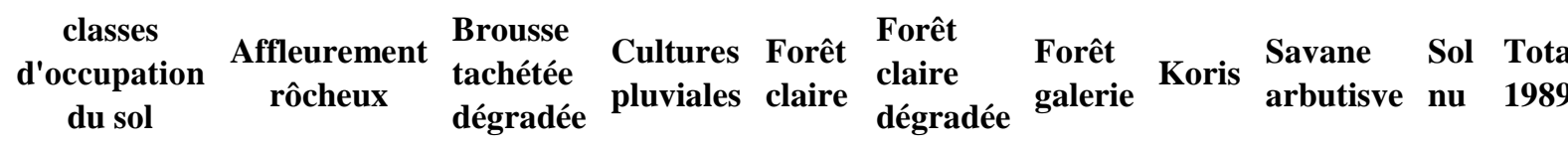

\begin{tabular}{|c|c|c|c|c|c|c|c|c|c|c|}
\hline $\begin{array}{l}\text { Affleurement } \\
\text { rôcheux }\end{array}$ & 1031 & - & - & - & - & - & - & - & - & 1031 \\
\hline $\begin{array}{l}\text { Brousse } \\
\text { tachétée } \\
\text { dégradée }\end{array}$ & - & 1508 & 3105 & - & - & 21 & & 5 & 26 & 4665 \\
\hline $\begin{array}{l}\text { Cultures } \\
\text { pluviales }\end{array}$ & - & & 8165 & - & - & - & - & 12 & 344 & 8521 \\
\hline Forêt claire & - & 1639 & 2681 & 16150 & 7423 & 91 & 51 & 18 & 113 & 28166 \\
\hline $\begin{array}{l}\text { Forêt claire } \\
\text { dégradée }\end{array}$ & - & 416 & 3033 & 338 & 20018 & 150 & - & - & 117 & 24072 \\
\hline Forêt galerie & - & - & 69 & & 504 & 4981 & 87 & - & 26 & 5667 \\
\hline $\begin{array}{l}\text { Savane } \\
\text { arbutisve }\end{array}$ & - & & 2974 & - & 2205 & - & - & 52 & - & 5043 \\
\hline Total & 1031 & 3563 & 20027 & 16488 & 30150 & 5243 & 138 & 87 & 440 & 77167 \\
\hline
\end{tabular}


Tableau 6 : Synthèse de l'évolution des différentes unités d'occupation entre 1975 et 2013.

\begin{tabular}{|c|c|c|c|c|c|c|c|}
\hline \multirow{2}{*}{$\begin{array}{l}\text { Type d'occupation des } \\
\text { sols }\end{array}$} & \multicolumn{2}{|c|}{ Etat en 1975} & \multicolumn{2}{|c|}{ Etat en 2013} & \multirow{2}{*}{$\begin{array}{l}\text { Progression } \\
\text { 1975-2013 } \\
\text { (ha) }\end{array}$} & \multirow{2}{*}{$\begin{array}{l}\text { Régression en } \\
\text { 1975-2013 } \\
\text { (ha) }\end{array}$} & \multirow{2}{*}{$\begin{array}{l}\text { Taux de } \\
\text { Changement en } \\
\text { 1975-2013 (\%) }\end{array}$} \\
\hline & ha & $\%$ & ha & $\%$ & & & \\
\hline Affleurement rôcheux & 1031 & 1,3 & 1031 & 1,3 & 0 & & 0,0 \\
\hline $\begin{array}{l}\text { Brousse tachetée } \\
\text { dégradée }\end{array}$ & 3214 & 4,2 & 2570 & 3,3 & & -644 & $-0,9$ \\
\hline Cultures pluviales & 4536 & 5,9 & 21802 & 28,3 & 17266 & & 6,5 \\
\hline Forêt claire & 43008 & 55,7 & 20601 & 26,7 & & -22407 & $-3,1$ \\
\hline Forêt claire dégradée & 18774 & 24,3 & 26651 & 34,5 & 7877 & & 1,5 \\
\hline Forêt galerie & 6604 & 8,6 & 696 & 0,9 & & -5908 & $-9,4$ \\
\hline Savane Arbustive & & 0,0 & 3423 & 4,4 & 3423 & & \\
\hline Koris & & 0,0 & 2 & 0,0 & 2 & & \\
\hline Sol nu & & 0,0 & 391 & 0,5 & 391 & & \\
\hline TOTAL & 77167 & 100 & 77167 & 100 & $\mathbf{0}$ & & \\
\hline
\end{tabular}

Tableau 7: Dynamique des unités d'occupation des sols en hectare dans la RTFT entre 1975 et 2013.

\begin{tabular}{|c|c|c|c|c|c|c|c|c|c|c|}
\hline $\begin{array}{l}\text { Unités d'occupation } \\
\text { des sols }\end{array}$ & $\begin{array}{l}\text { Affleuremen } \\
\text { t rocheux }\end{array}$ & $\begin{array}{l}\text { Br-*ousse } \\
\text { tachetée } \\
\text { dégradée }\end{array}$ & $\begin{array}{l}\text { Cultures } \\
\text { pluviales }\end{array}$ & $\begin{array}{l}\text { Forêt } \\
\text { claire }\end{array}$ & $\begin{array}{l}\text { Forêt } \\
\text { claire } \\
\text { dégradée }\end{array}$ & $\begin{array}{l}\text { Forêt } \\
\text { galerie }\end{array}$ & $\begin{array}{l}\text { Savane } \\
\text { arbustive }\end{array}$ & $\begin{array}{l}\text { Sol } \\
\text { nu }\end{array}$ & Koris & $\begin{array}{l}\text { Total } \\
1975\end{array}$ \\
\hline $\begin{array}{l}\text { Affleurement } \\
\text { rocheux }\end{array}$ & 1031 & - & - & - & - & - & - & - & - & 1031 \\
\hline $\begin{array}{l}\text { Brousse tachetée } \\
\text { dégradée }\end{array}$ & - & 179 & 3617 & & & & 68 & 38 & & 3904 \\
\hline Cultures pluviales & - & 1 & 1278 & 1484 & 1552 & 23 & 374 & 17 & & 4733 \\
\hline Forêt claire & - & 2390 & 8220 & $\begin{array}{l}1186 \\
1\end{array}$ & 13465 & 276 & 2921 & $\begin{array}{l}32 \\
0\end{array}$ & & 39456 \\
\hline $\begin{array}{l}\text { Forêt claire } \\
\text { dégradée }\end{array}$ & - & - & 1914 & 7255 & 11585 & 276 & 56 & 14 & & 21102 \\
\hline Forêt galérie & - & - & 6771 & 0 & 47 & 119 & 2 & $\mathbf{0}$ & 2 & 6944 \\
\hline \multicolumn{11}{|l|}{ Savane arbustive } \\
\hline \multicolumn{11}{|l|}{ Sol nu } \\
\hline \multicolumn{11}{|l|}{ Kori } \\
\hline Total 2013 & 1031 & 2570 & 21802 & $\begin{array}{l}2060 \\
1\end{array}$ & 26651 & 696 & 3423 & $\begin{array}{l}39 \\
1\end{array}$ & 2 & 77167 \\
\hline
\end{tabular}




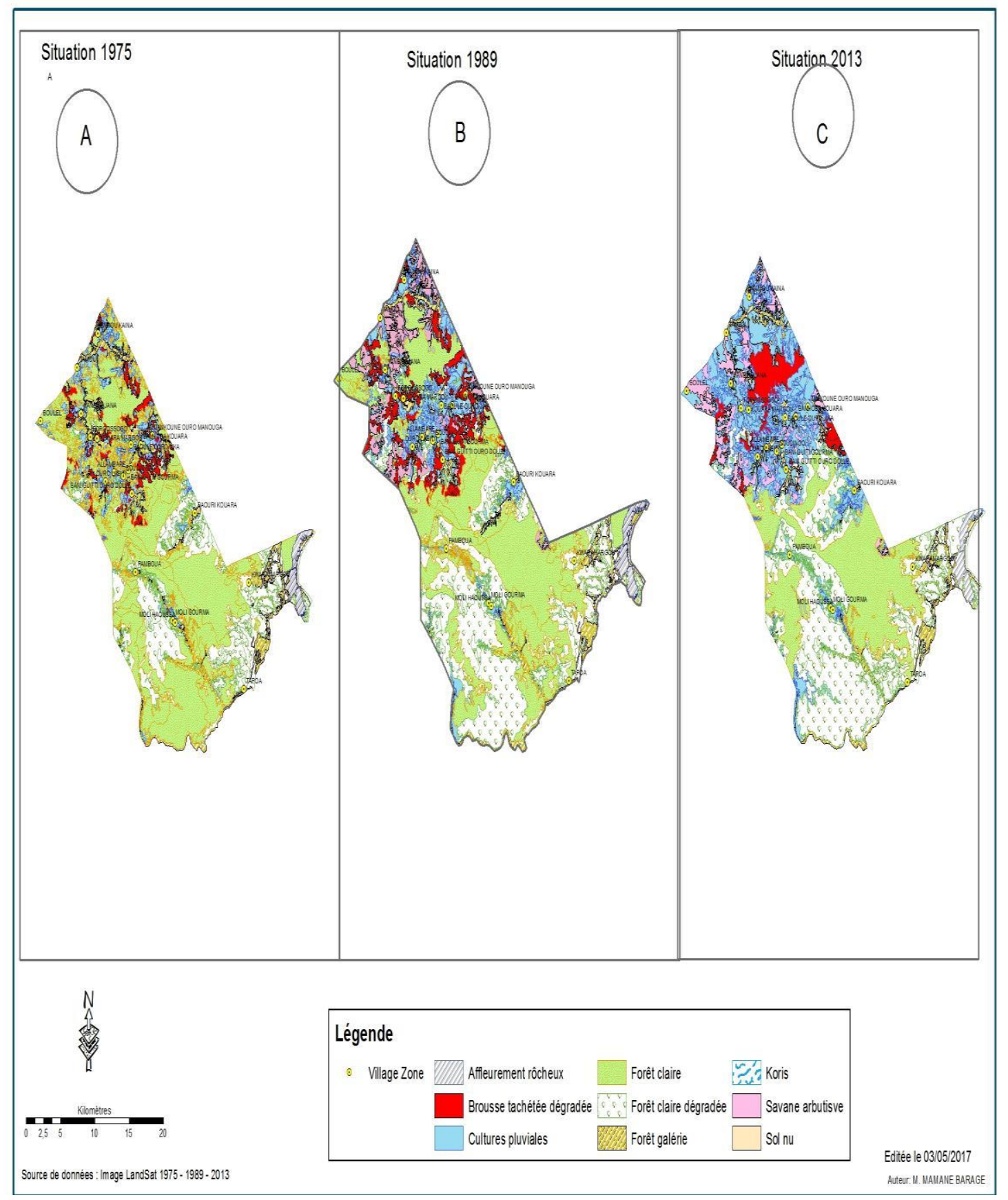

Figure 8: Evolution des zones de cultures entre 1975-2013 dans la Réserve Totale de Faune de Tamou. 


\section{DISCUSSION}

Il ressort de la comparaison des trois images TM de Landsat, avec une résolution relativement faible $(30 \mathrm{~m})$, une mesure de la dynamique du paysage dans la RTFT de Tamou de 1975 à 2013 (Tableau 4) avec plusieurs enseignements. En effet, deux aspects essentiels ont été démontrés :

\section{Impact anthropique}

L'analyse de la dynamique spatiotemporelle des unités d'occupation du sol de la RTFT du Parc W du Niger montre que les formations forestières font place de plus en plus aux formations anthropisées (champs, jachères anciennes et récentes, sol nu et Koris). Les résultats obtenus montrent l'ampleur et le rythme d'évolution des cultures pluviales sur les formations végétales naturelles entre 1975 et 2013 (4733 ha soit 6, $13 \%$ en 1975 qui atteignent 21802 ha soit $28,25 \% \quad 38$ ans après). Ces extensions agricoles sont très importantes dans la partie nord-ouest et le nord-est (Figure 8 C) car ces chiffes nous donnent une moyenne annuelle de 573 ha qui sont transformés en cultures pluviales.

Cette augmentation des surfaces de cultures pluviales est liée certainement à l'occupation anarchique de la RTFT cela a été soutenu par Harouna (2005) en confirmant que l'effectif de migrants du Zarmaganda est estimé en 1984 à 15000 personnes et à chaque phénomène de crise alimentaire ou de disette, la population de cette localité continue à migrer vers cette zone. Dans la RTFT avec les occupations humaines, les cultures pluviales sont plus concentrées au centre et au nord alors que dans la partie sud proche du Parc $\mathrm{W}$ où la réglementation n'autorise pas la création de villages (exception faite de celui de Moli Haoussa) la pression est faible. En effet, même l'existence des champs de culture dans cette partie (zone tampon) est le fait de la présence des gourmantchés burkinabés, populations ayant fui le noyau central en 1953 qui reviennent cultiver la terre sans véritablement créer un village (Souley, 2010b). Les conditions naturelles favorables (terres fertiles disponibles) en ont fait ces dernières années que la RTFT fait face à une forte pression agricole. Actuellement, certains agriculteurs audacieux défrichent des nouvelles terres sans autorisation. Quant aux éleveurs, ils exploitent les maigres ressources fourragères à prédominance de Zornia glochidiata. Ces résultats qui corroborent ceux d'Amadou (2005) et de Souley (2010c) obtenus dans la réserve de biosphère (incluant la RTFT, le parc national W et la zone girafe), présentent, pour la première fois, les transformations de l'occupation de terre dans la RTFT. Ces résultats sont conformes à l'analyse d'Ibro et Assoumane (2009) qui défendent que $66 \%$ de la superficie des forêts ont subi une dégradation, et $13 \%$ de cette dégradation sont dus à l'avancée des cultures pluviales dans la région de Tillabéry où se situe la RTFT. La même tendance est notée dans d'autres régions du Niger, telles que Diffa, Zinder, Maradi, où ROSELT-OSS (2009) et Abdourhamane et al (2012); ont montré que les formations denses, particulièrement les savanes boisées, sont les plus affectées par les activités anthropiques et les péjorations climatiques. Cependant ROSELT-OSS, (2009) conclut aussi à Torodi- Tondikandia (région de Tillabéry) que les changements les plus importants sont enregistrés au niveau des unités de cultures pluviales. Le même constat est effectué dans d'autres pays d'Afrique de l'Ouest notamment au Burkina et au Bénin, les études réalisées dans les périphéries de la Réserve de la Biosphère Transfrontalière du W (RBTW) par Orékan (2006b), Ouédraogo et al. (2010) et Julien et al. (2014), Boalidioa et al. (2015) concluent qu'il existe une forte corrélation entre la croissance démographique et la dégradation des ressources naturelles de la périphérie du parc W du Burkina et du Bénin. Des recherches menées sur la régression des formations forestières naturelles par Mama et al. (2013) dans la zone soudanienne au nord du Bénin, ainsi que celles de Bogaert et al. (2004), Diallo et al. (2011a) et Orékan (2007) corroborent nos résultats. En plus, dans le sud du Parc National du W au Bénin, 
Houessou et al. (2013) ont trouvé que l'agriculture est la principale force motrice de la dégradation de la couverture végétale dans cette région, corroborant ainsi nos résultats. Ainsi, le parc $\mathrm{W}$ se trouve affecté dans les trois pays (Niger, Bénin et Burkina Faso) par les mêmes forces que subissent les paysages au niveau de ces zones périphériques.

\section{L'impact climatique}

Depuis la fin des années 1970, la récession pluviométrique a été observée en Afrique de l'Ouest (Ozer et al., 2010a).

Cette situation est observée dans notre zone d'étude où l'analyse du graphique des anomalies standardisées des précipitations moyennes (Figure 3) révèle une variabilité climatique .Ainsi la décennie 1960 se caractérise par une pluviosité excédentaire par rapport à la normale 1961-1990 tandis que la pluviosité des décennies 70 et 80 est globalement déficitaire. A partir de la décennie 1990-2000 la pluviosité de la zone se caractérise par des années excédentaires mais souvent avec des années déficitaires. La décennie 2001-2011 est relativement plus humique que la précédente avec toutefois des épisodes secs de plus en plus longs, ce qui pourrait contribuer à expliquer l'état de régression des unités paysagères de la RTFT. Les entretiens avec les populations locales et les agents chargés de la gestion des aires protégées, ont confirmé que ces sècheresses, combinées aux actions anthropiques, ont contribué à la dégradation des ressources naturelles de la réserve.

Ces phénomènes sont inquiétants pour la gestion de la biodiversité et le fonctionnement des écosystèmes dans les aires protégées (Clerici et al., 2007b). En effet, en l'espace de 38 ans (1975 -2013), les unités d'occupation du sol de la RTFT ont connu d'importantes transformations (observation directe), de nombreuses mares, jadis permanentes tarissent le plus souvent en mars -avril mettant la faune à rude épreuve. De nombreux ligneux morts sur pied sont visibles surtout dans la partie nord et les espèces végétales les plus touchées sont, entre autres : Combretum micrantum, Combretum nigricans. Cette situation a aussi eu comme effet une diminution drastique des forêts galeries qui étaient 9\% en 1975 et $0,9 \%$ en 2013. Cependant, la contrainte climatique n'est pas simplement la rareté des précipitations, mais également leur variabilité, leur répartition et leur imprévisibilité qui influencent les écosystèmes sahéliens (Ozer et al., 2010b). Plusieurs études ont montré les impacts de la sécheresse sur la flore (Hulme et al., 2001 ). Certaines ont mis en évidence que les sécheresses répétées dans les années 1970 et 1980 ont eu directement pour effet, notamment en Afrique, l'augmentation de la mortalité des espèces ligneuses des écosystèmes sensibles (Inoussa et al., 2011b). D'autres ont montré que les sécheresses récurrentes ont rendu plus difficile la capacité de régénération naturelle. Pour les espèces herbacées, un changement a lieu dans la composition des espèces des aires de parcours qui se caractérise par la raréfaction, voire la disparition, de plantes pérennes telles que Andropogon gayanus .Il a été constaté que la végétation herbacées, jadis à dominantes de graminées vivaces, est passée à une végétation à composante mixte de plantes annuelles graminées (FAO, 2001b). Le prolongement des périodes de chaleur et l'assèchement des milieux induisent des stress physiologiques pour les arbres qui pourraient réduire ses systèmes de défense contre les attaques pathologiques (Wardell et al., 2003 cité par Diallo et al., 2011b). Aujourd'hui, dans la RTFT, les espèces les plus sensibles à la sècheresse disparaissent progressivement. Elles sont remplacées pour les ligneux par des espèces comme Acacia seyal, Guiera senegalensis, Combretum glutinosum (enquête terrain). Quant aux herbacées pérennes; elles sont remplacées par des herbacées annuelles comme Cenchrus biflorus et Sida cordifolia qui se sont progressivement installées sur les grandes étendues autrefois occupés par une pluralité d'espèces (FAO, 2001b). Des résultats similaires sont obtenus par d'autres auteurs qui montrent que les effets de la sécheresse en milieu de savane sont observés à travers les variations de la couverture ligneuse qui sont très 
indicatrices des changements climatiques à l'échelle locale (Koné et al., 2007b ; Diallo et al.,2011c ; Kouassi et al., 2012).

Pour faire face à cette menace qui pèse sur la RTFT, diverses actions sont à entreprendre. Dans le court terme d'une part, la mise en œuvre des activités régénératrices de revenus dans les villages riverains s'avère nécessaire, et d'autre part les agents des services déconcentrés de l'Etat chargés de la conservation de cette réserve doivent rendre plus opérationnels les principes de cogestion et de l'exercice des droits d'usage. En effet, l'intégration agriculture élevage foresterie peut être perçue comme une stratégie paysanne d'adaptation et/ou d'atténuation face aux risques socio-environnementaux (Soukaradji et al., 2017 ; Sitou et al., 2018). Ces auteurs soutiennent nos actions d'améliorations, en confirmant l'importance de cette intégration dans la région de Maradi au Niger.

En effet, au cours des enquêtes socioéconomiques, des besoins en valorisation des produits forestiers non ligneux (PFNL) ont été exprimés par les populations riveraines de la RTFT. Ces types d'activités peuvent être développés en vue de lutter efficacement contre la pauvreté extrême qui pousse certaines couches des populations de la zone à la surexploitation du bois qui est un des facteurs de la dégradation des ressources ligneuses de la réserve cité par les communautés locales. Ces dernières souhaitent aussi en cas d'infractions, l'application stricte de la réglementation en vigueur afin de permettre une prise de conscience collective sur l'importance de la conservation de la RTFT.

A long terme, un dispositif de suivi écologique et environnemental permettrait de suivre et de pallier à toutes les nouvelles situations.

\section{Conclusion}

L'étude diachronique des images Landsats de la RTFT a permis de mettre en évidence les différentes formes de conversions subies par les formations forestières des unités paysagères. Ainsi, on peut noter que pendant une durée de 38 ans (de 1975 à 2013), les unités d'occupation du sol de la réserve totale de faune de Tamou ont subi d'énormes perturbations.

En effet, la formation forestière qui était estimée à $92,78 \%$ de la superficie totale de la RTFT en 1975 s'est réduite à 68,54\% en 2013. Par ailleurs les superficies des cultures pluviales qui étaient estimées à 5,88\% en 1975 ont progressivement évolué pour atteindre $28,25 \%$ en 2013 de la superficie totale.

Outre cette montée du front agricole, on note l'apparition des sols nus et des koris dans la période de 1989 à 2013. Ces occupations des sols étaient pratiquement inexistantes entre 1975 et 1989. Ainsi, les formations forestières ont cédé progressivement leur place aux formations anthropisées. L'agriculture, les feux de brousses, le surpâturage les variations climatiques constituent les principales causes de conversion de ces unités paysagères.

Ces nouvelles unités apparues, notamment les terres de cultures, sont à leur tour exposées à la surexploitation, et deviennent de moins en moins productives. Les quelques mesures pour limiter cette évolution doivent par conséquent être renforcées.

\section{CONFLIT D'INTERETS}

Les auteurs de ce manuscrit déclarent qu'il n'y a aucun conflit d'intérêt entre eux.

\section{CONTRIBUTIONS DES AUTEURS}

Dans la réalisation de la présente étude, BM et GA ont élaboré le protocole de recherche, collecté et traité les données et aussi rédigé le manuscrit. MB et JMKA ont apporté des conseils dans la collecte et le traitement des données. Enfin MB, JC et JMKA ont participé à la relecture du document. Ils ont tous approuvé la version finale du manuscrit. 


\section{REFERENCES}

Abdourhamane H, Morou B, Mahamane A, Saadou M, Issaka A. 2012. Caractérisation de la dynamique spatiotemporelle de l'occupation des terres dans le complexe des forêts de classées de Dankada Dodo-Dan Gado (Région de Maradi, Niger). Journal des Sciences de l'Environnement, 1(1): 16-26. DOI: https://www.researchgate.net/publication 1275041880 .

Amadou B. 2005. Rapport scientifique, observatoire de Tamou, Réseau d' Observatoires de Surveillance Ecologique à Long Terme Niamey Niger $65 \mathrm{p}$.

Aubreville A. 1957. Accord de Yangambi sur la nomenclature des types africains de végétation. Bois et Forêts des Tropiques, 51 : 23-27.

Avakoudjo J, Mama A, Toko I, Kindomihou V, Sinsin B. 2014. Dynamique de l'occupation du sol dans le Parc National $\mathrm{du} \mathrm{W}$ et sa périphérie au nord-ouest du Bénin. Int. J. Biol. Chem. Sci., 8(6): 2608-2625. DOI http://dx.doi.org/10.4314/ijbcs.v8i6.22

Bachir M. 2013. Dynamique des services éco systémiques dans le bassin versant d'Oum Zessar (Sud Est tunisien), Mémoire de Master II, Institut national Agronomique de Tunisie, 102p.

Bamba I, Mama AD, Neuba FR, Koffi KJ, Traoré D, Visser M. 2008. Influence des actions anthropiques sur la dynamique spatio-temporelle de l'occupation du sol dans la province du Bas Congo(R.D.Congo). Sces. Nat., 5 : 4960. DOI: $10.4314 /$ scinat.v5i1.42151

Barbier N, Couteron P, Lejoly J, Deblauwe V, Lejeune O. 2006. Self-organised vegetation patterning as fingerprint of climate and humain impact on semiarid ecosystèmes. J. Ecol., 94-47. DOI: 10.1111/j.1365-2745.2006.01126.x

Bogaert J, Ceulemans R, Salvador-Van ED. 2004. Decision tree algorithm for detection of spatial processes in landscape transformation. Env. Manag, 33: 62-73. DOI: http://www.academia.edu/2168608
Bergaoui M, Alouini A. 2001. Caractérisation de la sécheresse météorologique et hydrologique : cas du bassin versant de Siliana en Tunisie. Sécheresse, 12: 20513. DOI: http:// www.secheresse.info/spip.php.article709 6

Clerici N, Bodini A, Eva H, Grégoire J M, Dulieu D, Paolini C. 2007. Increased Isolation of Two Biosphere Reserves and Surrounding Protected Areas (WAP Ecological Complex, West Africa). Journal for Nature Conservation, 15: 26-40. DOI:10.1016/j.jnc.2006.08.003

Diallo H, Bamba I, Barima YSS, Visser M, Ballo A, Mama A, Vranken I, Maiga M, Bogaert J. 2011. Effets combinés du climat et des pressions anthropiques sur la dynamique évolutive de la dégradation d'une aire protégée du Mali (la Réserve de Fina, Boucle du Baoulé). Sécheresse, 22(2): 97-107. DOI : http://www .jle.com/download/sec288975.

Démocratie 2000. 2004. Rapport Etude socio-foncière dans la zone Ayinoma canton de Tamou, département de Say, p. 47.

De Wispelaere G. 2002. Cartographie de la Végétation du Complexe " $W »$. Cirad éditions : Montpellier.

Essifi B. 2008. Etude comparative des risques d'érosion autour des points d'eau dans les parcours du Dahar et d'El Ouara dans la région de Tataouine : Application de la télédétection et des SIG, mémoire de master spécialisé en géomatique, Faculté des lettres des arts et des Humanités de Manouba /institut des Régions Arides de Médinine, P.122.

FAO (Food and Agriculture Organization). 1996. Forest resources assessment, Survey of tropical forest cover and study of change processes, forestry Paper, 130. FAO: Roma, 152.

FAO (Food and Agriculture Organization). 2001. La situation des ressources génétiques forestières de la zone sahélienne et nord-soudanienne, plan d'action sous-régional, note thématique. 
FAO : Rome, 18 .

Giannini A, Saravanan R, Chang P, 2003. Oceanic forcing of Sahel rainfall on interannual to interdecadal time scales. Sciences, 303:1027-1030. DOI: 10.1126/science. 1089357

Harouna M. 2005. Rapport d'étude sur la migration de colonisation agricole et dynamique territoriales dans les communes rurales de Say et de Tamou. Université Abdou Moumouni de Niamey, p.15 .

Houessou LG, Téka O, Toko II, Lykke AM, Sinsin B. 2013. Land use and land cover change at "W" Biosphere Reserve and its surroundings areas in Benin Republic (West Africa). Env. Nat. Res. Resear., 3(2):

87-101.

DOI:10.5539/enrr.v3n2p87.

Hulme M. 2001. Climatic perspectives on Sahelian desiccation: 1973-1998. Global Environmental Change, 11(1): 19-29. DOI :https://www.sciencebase.gov

Ibro A, Assoumane G. 2009. Occupation des sols des forêts classées du Niger et l'analyse des dynamiques de changement, Programme d'évaluation des ressources forestières $\mathrm{FAO}, 25 \mathrm{p}$.

INS (Institut National de la Statistique). 2012. Présentation du quatrième résultat du recensement générale de la population et l'Habitat (RGP/H) Niger, $15 \mathrm{p}$.

Inoussa MM, Ali M, Cheikh M, Mamane S, Bachmann Y. 2011. Dynamique spatiotemporelle des forets claires dans le Parc national du W du Niger (Afrique de l'Ouest) 9 p. Sécheresse, 22 :108-116. DOI : $10.1684 / \mathrm{sec} .2011 .0305$

KONÉ M, Aman A, Adou Yao CY, Coulibaly L, N'guessan KE. 2007. Suivi diachronique par télédétection spatiale de la Couverture ligneuse en milieu de savane soudanienne en Côte d'Ivoire. Revue Télédétection, 7(1-2-3-4): 433446. DOI : http://docplayer.fr/30666741

Kouassi AM, Kouamé KF, Ahoussi KE, Oularé S, Biemi J. 2012. Impacts conjugués des changements climatiques et des pressions anthropiques sur les modifications de la couverture végétale dans le bassin versant du N'zi-Bandama (Côte d'Ivoire). Rev. Ivoir. Sci. Technol., 20: 124-146. DOI : http://www.revist.ci
Koffi DK, Tchaa B, Thiou TKT. 2016. Quantification des changements de l'occupation du sol dans la préfecture de Yoto (Sud - est Togo) à l'aide de l'imagerie satellitaire Landsat. Rev. Sc. Env. Lomé Togo., 13. DOI: https://hal.archives-ouvertes.fr/hal01409418

Lawali S, Diouf A, Morou B, Abdou Kona K, Saidou L, Guero C, Mahamane A. 2018. Régénération Naturelle Assistée (RNA) : outil d'adaptation et résilience des ménages ruraux d'Aguié au Niger. Int. $J$. Biol. Chem. Sci., 12(1): 75-89. DOI: https://dx.doi.org/10.4314/ijbcs.v12i1.6

Lillesand TM, Kiefer RW.1994. Remote Sensing and Image Interpretation. John Wiley and Sons, Inc: Hoboken, $750 \mathrm{p}$.

Mama A. 2013. Anthropisation des paysages au Bénin : dynamique, fragmentation et développement agricole. Thèse de Doctorat, Université Libre de Bruxelles, Belgique, p.198.

Maman MI, Ali M, Anne ML, Abassa I, Mahamane S . 2011. Cartographie et description des types de vegetation au parc national du W du Niger 22, 3. DOI : $10.1684 / \mathrm{sec} .2007 .0101$

Ministère de l'environnement et de la lutte contre la désertification. 2001. Nomenclature pour la construction de bases de données sur l'occupation des sols (NOS) au Niger au Sud du 16 ème parallèle. $69 \mathrm{P}$.

Noyola MC, Mering C, Rojas B A. 2009. Evaluation du changement de l'occupation du sol à l'aide des images Landsat et Spot : Champ volcanique de la Sierra Chichinautzin (Mexique). 24th Internacional Cartography Conference: The World's Geo-Spatial Solutions (ICC, 2009). 12p. https://icaci.org/files/documents/ICC_pro ceedings/ICC2009/html/refer/6_2.pdf

Ouédraogo I, Tigabu M, Savadogo P, Compaore H, Oden PC, Ouadba JM. 2010. Land cover change and its relation with population dynamics in Burkina faso, West Africa. Land Degrad. Dev., 21 : 453-462 . DOI : https://doi.org/10.1002/ldr.981

Orékan V, Tente B, Houndagba CJ, Abdoulaye D . 2006. Occupation des 
terres et dynamique du couvert végétal. Atlas de IMPETUS, chapitre utilisation des terres, 153-162.

Orékan V A. 2007. Implémentation du modèle local CLUE-s aux transformations spatiales dans le Centre Bénin aux moyens de données socio-économiques et de télédétection. Thèse de doctorat, Université de Bonn, Bonn, Suisse, p.204.

Oloukoi J, Mama VJ, Agbo F. 2006. Modélisation de la dynamique de l'occupation des terres dans le département des collines au Bénin. Télédétection, 6(4) : 305-323. DOI :

https://www.researchgate.net/publication 1279851907.

Orékan V, Abdoulaye D, Houndagba C, Sinsin B . 2014. Dynamique prospective des écosystèmes forestiers classés au Bénin: quelle perspective pour une utilisation durable. http://.www.bj.chmcbd.net > RESSOURCES > Publications , Flore ,

Ozer P, Hountondji YC, Niang AJ, Karimoune S, Manzo OL, Salmon M. 2010. Désertification au Sahel: historique et perspectives. BSGLg., 54: 69-84. DOI:

https://popups.uliege.be:443/07707576/index.php?id=942.

ROSELT (Réseau d'Observatoires pour la Surveillance Ecologique à Long Terme). 2009a. DNSE DU Niger : Etat de référence des observations de Diffa, Zinder, Azawad et Torodi-Tondikandia.
Au Niger 131p.

Soukaradji B, Abdou A, Lawali S, Aboubacar I, Mahamane A, Saadou M. 2017. Typologie des exploitations agricoles familiales : cas de la périphérie de la forêt protégée de Baban Rafi du Niger. Int. J. Biol. Chem. Sci., 11(3): 10961112.

DOI: http://dx.doi.org/10.4314/ijbcs.v11i3.14

Souley K. 2010a. Analyse des grands flux de migration vers la réserve et de leurs impacts sur la communauté locale à la périphérie du parc W du Niger. Thèse de doctorat, Université Abdou Moumouni de Niamey Niger, p. 440.

Sarr MA. 2009. Cartographie des changements de l'occupation du sol entre 1990 et 2002 dans le nord du Sénégal(Ferlo) à partir des images Landsat, Cypergeo: European Journal of Géographie (en ligne), Environnement, Nature, paysage, article 472 , mis en ligne le 07 octobre 2009, consulté le 16 janvier 2015 : URL :http ://cybergeo.revues.org/22707; DOI :104000/cypergeo.22707.

Tankoano MB, Hien M, Dibi NH, Sanon MZ, Master, Yameogo JT, Somda I. 2015 Dynamique spatio-temporelle des savanes boisées de la forêt classée de Tiogo au Burkina Faso. Int. J. Biol. Chem. Sci., 9(4): 1983-2000. DOI: http://dx.doi.org/10.4314/ijbcs.v9i4.23 